\title{
Multiscale Weighted Ensemble Kalman Filter for Fluid Flow Estimation
}

\author{
Sai Gorthi, Sébastien Beyou, Thomas Corpetti*, and Etienne Mémin \\ INRIA / FLUMINANCE, 35042 Rennes Cedex, France \\ CNRS/LIAMA, PO Box 2728, Beijing 100190, PR China \\ \{sai.gorthi, sebastien.beyou, thomas.corpetti, etienne.memin\}@inria.fr \\ http://www.irisa.fr/fluminance/indexFluminance.html
}

\begin{abstract}
This paper proposes a novel multi-scale fluid flow data assimilation approach, which integrates and complements the advantages of a Bayesian sequential assimilation technique, the Weighted Ensemble Kalman filter (WEnKF) [12], and an improved multiscale stochastic formulation of the Lucas-Kanade (LK) estimator. The proposed scheme enables to enforce a physically plausible dynamical consistency of the estimated motion fields along the image sequence.
\end{abstract}

\section{Introduction}

The analysis of geophysical fluid flows is of the utmost importance in domains such as oceanography, hydrology or meteorology for applications of forecasting, studies on climate changes, or for monitoring hazards or events. In all these domains orbital or geostationary satellites provide a huge amount of image data with a still increasing spatial and temporal resolution. Compared to in situ measurements supplied by dedicated probes or Lagrangian drifters, satellite images provide a much more denser observation field. They however offer only an indirect access to the physical quantities of interest, and give rise consequently to difficult inverse problems to estimate characteristic features of the flow such as velocity fields or vorticity maps.

Fluid motion estimation techniques differ mainly on the smoothness prior they are handling: first order penalization[13], second order div-curl regularization $[2,14]$, or power law auto-similarity principles [6]. These methods provide accurate instantaneous displacements, however they may exhibit difficulties for mid to small scales measurements due to the smoothing prior used and photometric uniform regions. All these difficulties may thus generate inconsistent measurements along time. For interested readers, a complete overview of fluid motion estimation techniques can be found in [7].

Dynamical consistency of the velocity measurements can be enforced by embedding the estimation problem within an image based assimilation process. Variational assimilations of image information have been recently considered for the estimation of fluid motion fields $[1,11]$. Those methods, though efficient, constitutes batch methods, which requires forward and backward integrations of the dynamical system and the associated tangent linear dynamics respectively. 
The latter relies implicitly on a linearization of the dynamics and is adapted in practice for short time horizon.

On the other hand, stochastic filters are also well known techniques for data assimilation. Recently, a data assimilation procedure embedding an Ensemble Kalman filter (EnKF) [5] into the particle filter (PF) framework, referred to as Weighted Ensemble Kalman filter (WEnKF), has been proposed [12]. This filter has shown to be efficient on toy examples with synthetic measurements. The objective of this work consists to specify such a procedure from local noisy velocity measurements and their uncertainties.

\section{Stochastic Lucas-Kanade Estimator}

This section first presents a stochastic formulation of the well known LucasKanade (LK) optical flow approach [9] that will be used to provide local motion measurements in the assimilation method we propose. This technique departs somewhat from the traditional Lucas and Kanade motion estimator. It leads naturally to a continuous multiresolution formulation and enables not only to extract the motion fields at different resolutions but supplies uncertainties of those estimates as well.

In what follows, we represent the image luminance with $f$, and a grid of $2 \mathrm{D}$ points $\mathbf{X}=\left(X^{1}, \ldots, X^{n}\right)^{T} \in R^{2 n}$, represents the grid point locations. The image over a regular grid at time $t-1, I=f\left(\mathbf{X}_{t-1}, t-1\right)$, is driven by the velocity field $\mathbf{v}\left(\mathbf{X}_{t-1}, t-1\right)$ to generate new point positions $\mathbf{X}_{t}$ at time $t$.

\subsection{Luminance variation with uncertainties}

In a stochastic formulation, if we assume that the $2 \mathrm{D}$ grid from $\mathbf{X}_{t-1}$ to $\mathbf{X}_{t}$ is transported by a velocity field, $\mathbf{v}$, up to a Brownian motion $\mathbf{B}_{t}=\left(B_{t}^{1}, \ldots, B_{t}^{n}\right) \in$ $R^{2 n}$, we can write: $d \mathbf{X}_{t}=\mathbf{v}\left(\mathbf{X}_{t-1}, t-1\right) d t+\Sigma\left(\mathbf{X}_{t}, t\right) d \mathbf{B}_{t}$, here $\Sigma$ is the covariance matrix and $d \mathbf{X}_{t}=\mathbf{X}_{t}-\mathbf{X}_{t-1}$. Assuming uncorrelated uncertainties with local isotropic standard deviation $\sigma\left(\mathbf{X}_{t}, t\right)$, the noise term reads $\Sigma\left(\mathbf{X}_{t}, t\right) d \mathbf{B}_{t}=$ $\operatorname{diag} \sigma\left(\mathbf{X}_{\mathrm{t}}, \mathrm{t}\right) \otimes \mathcal{I}_{2} \mathrm{~d} \mathbf{B}_{\mathrm{t}}, \mathcal{I}_{2}$ being the $2 \times 2$ identity matrix, and $\otimes$ denoting the Kronecker product.

The differential of luminance function $f$ defined for each spatial point at time $t$ is obtained through stochastic calculus differentiation using the celebrated Ito formulae [10] as :

$$
d f\left(\mathbf{X}_{t}, t\right)=\frac{\partial f\left(\mathbf{X}_{t}, t\right)}{\partial t} d t+\sum_{i=(1,2)} \frac{\partial f\left(\mathbf{X}_{t}, t\right)}{\partial x_{i}} d \mathbf{X}^{i}+\frac{1}{2} \sum_{(i, j)} \frac{\partial^{2} f\left(\mathbf{X}_{t}, t\right)}{\partial x_{i} \partial x_{j}} d\left\langle\mathbf{X}_{t}^{i}, \mathbf{X}_{t}^{j}\right\rangle
$$

The quadratic variation terms $d\left\langle\mathbf{X}_{t}^{i}, \mathbf{X}_{t}^{j}\right\rangle$ are computed based on the properties:

$$
d\left\langle B^{i}, B^{j}\right\rangle=d t ;\langle h(t), h(t)\rangle=\left\langle h(t), B^{i}\right\rangle=\left\langle B^{j}, h(t)\right\rangle=0 .
$$

With the considered uncertainties this yields a luminance variation:

$$
d f\left(\mathbf{X}_{t}, t\right)=\left(\frac{\partial f}{\partial t}+\nabla f^{T} \mathbf{v}+\frac{1}{2} \sigma^{2} \Delta f\right) d t+\sigma \nabla f^{T} d \mathbf{B}_{t}
$$


The operators $\nabla$ and $\Delta$ represents the $2 \mathrm{D}$ gradient and Laplacian of the luminance function, respectively. This model obviously comes back to the standard brightness consistency assumption for zero uncertainties $(\sigma=0)$. Interest readers may refer to [3] for a more complete presentation of this estimator.

\subsection{Data model with uncertainties and local estimation}

In a minimum least square sense, we define the motion to be estimated $\mathbf{v}\left(\mathbf{X}_{t-1}, t-\right.$ 1 ), as the minimum conditional variance of the luminance variation. Starting from known grid $\mathbf{X}_{t-1}$, it can estimated by minimizing the expectation $\mathbb{E}\left(d f^{2}\left(\mathbf{X}_{t}, t\right) / \mathbf{X}_{t-1}\right)$. This conditional expectation given $\mathbf{X}_{t-1}$ of a function of a stochastic processes $\mathbf{X}_{t}$ driven by an Ito diffusion (1) discretized through an Euler-Maruyama scheme, $\mathbf{X}_{t}=\mathbf{X}_{t-1}+\mathbf{v}\left(\mathbf{X}_{t-1}, t-1\right) d t+\Sigma^{1 / 2}\left(\mathbf{B}_{t+1}-\mathbf{B}_{t}\right)$, can be expressed as the following convolution: $\mathbb{E}\left(d f^{2}\left(\mathbf{X}_{t}, t\right) / \mathbf{X}_{t-1}\right)=d f^{2}\left(\mathbf{X}_{t-1}+\right.$ $\mathbf{v}, t) \star g_{\Sigma}$, where $g_{\Sigma}=N(0, \Sigma)$ is a multidimensional zero mean Gaussian. From the illumination variation equation $(1)$, the cost function to be minimized reads hence:

$$
\mathcal{H}(f, \mathbf{v})=g_{\Sigma} \star\left(\frac{\delta f}{\delta t}+\nabla f \cdot \mathbf{v}+\frac{1}{2} \sigma^{2} \Delta f\right)^{2} .
$$

To alleviate the ill-posed nature of $(2)^{1}$ we assume a locally constant flow within a Gaussian window of variance $\lambda^{\ell}$ centered at location $(x, y)$ as in the standard Lucas-Kanade estimator. At point $(x, y)$, the estimate $\mathbf{v}$ should hence minimize:

$$
\arg \min _{v} g_{\lambda^{\ell}} \star \mathcal{H}(f, \mathbf{v})
$$

Differentiating (3) with respect to $\mathbf{v}$ and equating to zero, at any position $(x, y)$ (with $f_{x}, f_{y}, f_{t}$ representing the spatial $(x, y)$ and temporal derivatives of $f$ ) yields:

$$
\left(g_{\lambda^{\ell}} \star g_{\Sigma} \star\left[\begin{array}{cc}
f_{x}^{2} & f_{x} f_{y} \\
f_{x} f_{y} & f_{y}^{2}
\end{array}\right]\right) \mathbf{v}=-g_{\lambda^{\ell}} \star g_{\Sigma} \star\left(\frac{1}{2} \sigma^{2} \Delta f+f_{t}\right)\left[\begin{array}{l}
f_{x} \\
f_{y}
\end{array}\right] .
$$

\subsection{Multiresolution Analysis and Uncertainty estimation}

A multi-resolution analysis (formulated within an incremental framework) of this stochastic formulation can be accomplished by a coarse-to-fine decrease of the variance parameter associated to the local smoothing Gaussian window, $\lambda^{\ell}$, in (4). Furthermore, the quadratic variation of luminance function between $t-1$ and $t$ can be written as

$$
d\left\langle f\left(\mathbf{X}_{t}, t\right), f\left(\mathbf{X}_{t}, t\right)\right\rangle=\sigma^{2}\left\|\nabla f\left(\mathbf{X}_{t-1}+\mathbf{v}\left(\mathbf{X}_{t-1}, t\right), t\right)\right\|^{2} .
$$

In a probabilistic sense the variance parameters, in (5) can be estimated as:

$$
\sigma=\sqrt{\frac{\mathbb{E}\left(f\left(\mathbf{X}_{t-1}+\mathbf{v}\left(\mathbf{X}_{t-1}, t\right), t\right)-f\left(\mathbf{X}_{t-1}, t\right)\right)^{2}}{\mathbb{E}\left(\left\|\nabla f\left(\mathbf{X}_{t-1}+\mathbf{v}\left(\mathbf{X}_{t-1}, t\right), t\right)\right\|^{2}\right)}} \text { a.s. }
$$

This provides us a spatial distribution of the motion estimate uncertainties.

\footnotetext{
${ }^{1}$ Single equation, with two unknown components.
} 


\section{Monte Carlo implementation of stochastic filtering with the Weighted-Ensemble Kalman Filter}

In this section we briefly review the main principles driving the construction of the Weighted Ensemble Kalman filter, proposed in [12], and discuss its advantages and limitations in the context of fluid flow analysis. This technique is a particle implementation of a nonlinear stochastic filtering problem build upon an ensemble Kalman update stage. In the following section we recall briefly the basic elements constituting such filter.

\subsection{Stochastic filtering, filtering distribution}

Stochastic filters aim at estimating the posterior probability distribution $p\left(\mathbf{x}_{0: k} \mid \mathbf{y}_{1: k}\right)$ of a state variable trajectory $\mathbf{x}_{0: k}$ starting from an initial state $\mathbf{x}_{0}$ up to the state at the current time $\mathbf{x}_{k} \in \mathcal{R}^{n}$ given a complete measurements trajectory $\mathbf{y}_{1: k}$. The state variable trajectory is obtained through the integration of a dynamical system:

$$
\mathbf{x}_{t}=M\left(\mathbf{x}_{t-\delta t}\right)+\boldsymbol{\eta}_{t},
$$

where $M$ denotes a deterministic linear/nonlinear dynamical operator, corresponding to a discrete representation (through numerical integration with time step $\delta t$ ) of a physical conservation law describing the state evolution. And $\boldsymbol{\eta}_{t}$ is usually a white Gaussian noise of covariance $Q_{\delta t}$, that accounts for the uncertainties in the deterministic state model. However, as the true initial state is unknown, observation $\mathbf{y}_{k} \in \mathcal{R}^{m}$ of the state occurring at discrete instants are assumed to be available. These observations and the state variable are linked through:

$$
\mathbf{y}_{k}=H\left(\mathbf{x}_{k}\right)+\gamma_{k},
$$

a measurement equation where $\gamma_{k}$, the observation noise, is a white Gaussian noise with covariance matrix $R$, and $H$ stands for the linear/nonlinear mapping from the state variable space to the observation space. We note that the (integration) time step used for the state variable dynamics $\delta t$ is usually much smaller (about 10-100 times), than the latency $\delta k$ between two subsequent measurements. A sequence of measurements or observations from time 1 to $\mathrm{k}$ will be denoted by a set of vectors of dimension $m$ as: $\mathbf{y}_{1: k}=\left\{\mathbf{y}_{i}, i=1, \ldots, k\right\}$ where the latency between two successive measurements is arbitrarily set to $\delta k=1$.

A recursive expression of the filtering distribution $p\left(\mathbf{x}_{0: k} \mid \mathbf{z}_{1: k}\right)$, describing the distribution of the hidden Markov process we want to estimate conditioned upon the whole set of past observations $\mathbf{z}_{1: k}$, can be obtained from Bayes' law and the assumption that the measurements depends only on the current state:

$$
p\left(\mathbf{x}_{0: k} \mid \mathbf{y}_{1: k}\right)=p\left(\mathbf{x}_{0: k-1} \mid \mathbf{y}_{1: k-1}\right) \frac{p\left(\mathbf{y}_{k} \mid \mathbf{x}_{k}\right) p\left(\mathbf{x}_{k} \mid \mathbf{x}_{k-1}\right)}{p\left(\mathbf{y}_{k} \mid \mathbf{y}_{1: k-1}\right)} .
$$




\subsection{Linear Gaussian models and the Kalman Filter}

For a Gaussian initial distribution, linear dynamics and linear measurement operator, denoted by $\mathbf{M}$ and $\mathbf{H}$ respectively, the distribution $p\left(\mathbf{x}_{k} \mid \mathbf{y}_{1: k}\right)$ remains a Gaussian distribution whose first and second moment, $\mathbf{x}_{k}^{a}=\mathbb{E}\left(\mathbf{x}_{k} / \mathbf{y}_{1: k}\right)$ and $\mathbf{P}_{k}^{a}=\mathbb{E}\left(\left(\mathbf{x}-\mathbf{x}_{k}^{a}\right)\left(\mathbf{x}-\mathbf{x}_{k}^{a}\right)^{T} / \mathbf{y}_{1: k}\right)$, can be explicitly computed from the well known recursive Kalman equations [8]:

$$
\mathbf{x}_{k}^{f}=\mathbf{M} \mathbf{x}_{k-1}^{a}, \quad \mathbf{P}_{k}^{f}=\mathbf{M P}_{k-1}^{a} \mathbf{M}^{T}+\mathbf{Q}_{k},
$$

and

$$
\mathbf{K}_{k}=\mathbf{P}_{k}^{f} \mathbf{H}^{T}\left(\mathbf{H P}_{k}^{f} \mathbf{H}^{T}+\mathbf{R}\right)^{-1}, \mathbf{x}_{k}^{a}=\mathbf{x}_{k}^{f}+\mathbf{K}_{k}\left(\mathbf{y}_{k}-\mathbf{H} \mathbf{x}_{k}^{f}\right), \mathbf{P}_{k}^{a}=\left(\mathbb{I}-\mathbf{K}_{k} \mathbf{H}\right) \mathbf{P}_{k}^{f},
$$

here superscripts $f$ and $a$ on state variable and covariance denote the respective quantities before and after analysis (update) at time $k$, respectively. The prediction or forecast step (10) brings forward the first two moments of the state vector, from its previous time step $k-1$, through the dynamical model parameters, while the analysis or the correction step (11) provides the first two moments of the state characterizing the Gaussian filtering distribution at time $k$. The matrix $\mathbf{K}_{k}$ is referred to as the Kalman gain matrix.

\subsection{Particle implementation of the nonlinear filtering}

For nonlinear dynamics or nonlinear measurement equation a direct sampling from the filtering distribution is impossible since it would require the complete knowledge of the filtering distribution - which is in the general case a non Gaussian multimodal distribution - at a previous time.

Particle filtering techniques introduce a discrete approximation of the sought density as a sum of $N$ weighted Diracs:

$$
p\left(\mathbf{x}_{0: k} \mid \mathbf{y}_{1: k}\right) \approx \sum_{i=1}^{N} w_{k}^{(i)} \delta_{\mathbf{x}_{0: k}}\left(\mathbf{x}_{0: k}\right),
$$

centered on hypothesized locations of the state space sampled from a proposal distribution $\pi\left(\mathbf{x}_{0: k} \mid \mathbf{z}_{1: k}\right)$ (also called the importance distribution) approximating the true filtering distribution. Each sample is then weighted by a weight, $w_{k}^{(i)}$, accounting for the ratio between the two distributions. Any importance function can be chosen (with the only restriction that its support contains the filtering distribution one). Under weak hypotheses the importance ratio can be recursively defined as:

$$
w_{k}^{(i)} \propto w_{k-1}^{(i)} \frac{p\left(\mathbf{y}_{k} \mid \mathbf{x}_{k}^{(i)}\right) p\left(\mathbf{x}_{k}^{(i)} \mid \mathbf{x}_{k-1}^{(i)}\right)}{\pi\left(\mathbf{x}_{k}^{(i)} \mid \mathbf{x}_{0: k-1}^{(i)}, \mathbf{y}_{1: k}\right)} .
$$

By propagating the particles from time $k-1$ through the proposal density $\pi\left(\mathbf{x}_{k}^{(i)} \mid \mathbf{x}_{0: k-1}^{(i)}, \mathbf{y}_{1: k}\right)$, and by weighting the sampled states with $w_{k}^{(i)}$, a sampling of 
the filtering law is obtained. When the proposal distribution is set to the prior, the weights updating rule (13) simplifies to the data likelihood $p\left(\mathbf{y}_{k} \mid \mathbf{x}_{k}^{(i)}\right)$. This particular instance of the particle filter is called the Bootstrap filter and constitutes the most common filtering method based on particle filter. Nevertheless, such an importance function does not take into account the current observation and depends only weakly on the past data through the filtering distribution estimated at the previous instant. High dimensional probability distribution spaces being excruciatingly difficult to sample, it is very important to devise an importance function that enables focusing on the most meaningful areas of the state space. To that end it is essential to consider proposal distributions that take into account more significantly the past and current measurements. Along this idea, the weighted ensemble Kalman filter defines the proposal distribution from the sampling mechanisms of ensemble Kalman filtering techniques.

\subsection{Ensemble Kalman Filtering}

The Ensemble Kalman filter [4] can be interpreted as a Monte Carlo implementation of the Kalman filter recursion for the propagation of the two first moments. The Ensemble filter relies hence intrinsically on a Gaussian approximation of the filtering distribution.

More precisely, let us assume that we have sampled $N$ members from initial filtering distribution $p\left(\mathbf{x}_{0} / \mathbf{y}_{0}\right)$, denoted by $\mathbf{x}_{0}^{(i)}, i=1, \ldots, N$. Propagating these samples, iteratively, through the Kalman prediction and correction steps, provides us the Gaussian approximations of the prediction and filtering distributions.

The prediction step consists in propagating the ensemble members $\mathbf{x}_{k-1}^{a,(i)}$ and their associated uncertainties (noise) through the state dynamics in order to obtain a predicted particles or forecast ensemble as:

$$
\mathbf{x}_{k}^{f,(i)}=\sum_{t=k-1}^{k-\delta t}\left(M\left(\mathbf{x}_{t}^{f,(i)}\right)+\boldsymbol{\eta}_{t+\delta t}^{(i)}\right), \quad \mathbf{x}_{k-1}^{f,(i)}=\mathbf{x}_{k-1}^{a,(i)} .
$$

From this, the empirical mean, $\overline{\mathbf{x}}_{k}^{f}$, of the forecast ensemble and the corresponding empirical forecast covariance matrix $\mathbf{P}_{k}^{f_{e}}$ are computed. Using this ensemble based forecast covariance, an ensemble based Kalman gain matrix $\mathbf{K}_{k}^{e}$ can be computed. With this Kalman gain and the observation model the forecast ensemble members are then corrected towards the current observation.

This correction consists to update the forecast ensemble members $\mathbf{x}_{k}^{f,(i)}$, through the Kalman update equations, with a set of perturbed observation $\mathbf{y}_{k}+\gamma_{k}^{(i)}$ obtained from samples of the observation noise $\left\{\gamma_{k}^{(i)}, i=1, \ldots, N\right\}$. This provides an analysis ensemble members $\left\{\mathbf{x}_{k}^{a,(i)}, i=1, \ldots, N\right\}$ defined as:

$$
\mathbf{x}_{k}^{a,(i)}=\mathbf{x}_{k}^{f,(i)}+\mathbf{K}_{k}^{e}\left(\mathbf{y}_{k}+\gamma_{k}^{(i)}-\mathbf{H} \mathbf{x}_{k}^{f,(i)}\right) .
$$

Here, we note that, in the Kalman gain or in the update stage, computation of the high dimensional covariance matrix or inverse of the $n \times n$ covariance term, 
$\left(\mathbf{H P}_{k}^{f_{e}} \mathbf{H}^{T}+R\right)^{-1}$, are never explicitly computed nor stored. Rather, Kalman gain and update are efficiently implemented by defining and employing matrices with ensemble of perturbations. In most of the geophysical applications, the state vector related usually to temperature, pressure or velocity fields is of much higher dimension than the number of samples used in EnKF $N$. i.e., $n>>N$, thus, handling the perturbation matrices (instead of the actual corresponding covariance matrices) approximately brings down the number of operations from $O\left(n^{2}\right)$ to $O(n N)$. The inverse needed in the Kalman gain can be efficiently computed through the singular value decomposition of a $n \times N$ matrix [5].

\subsection{Weighted EnKF}

Starting from the descriptions of the previous section, a hybrid filtering procedure that takes advantage of both the particle filter and the EnKF can be devised. We briefly describe the approach proposed in [12].

The importance sampling principle indicates that a wide range of proposal distributions can be considered. We will experimentally show that a proposal distribution defined by the EnKF procedure constitutes an efficient proposal mechanism for particle filter techniques in high dimensional spaces.

Relying on the usual assumption of the EnKF (i.e. considering the dynamics as a discrete Gaussian system), the conditional distribution $p\left(\mathbf{x}_{k} \mid \mathbf{x}_{k}^{(i)}, \mathbf{y}_{k}^{o}\right)$ can be approached by a Gaussian distribution of respective mean and covariance [12]:

$$
\overline{\boldsymbol{\mu}}_{k}^{(i)}=\left(\mathbb{I}-\mathbf{K}_{k}^{e} \mathbf{H}\right) \sum_{t=k-1}^{k-\Delta t} M\left(\mathbf{x}_{t}^{f,(i)}\right)+\mathbf{K}_{k}^{e} \mathbf{y}_{k}^{o}, \quad \boldsymbol{\Sigma}_{k}^{e}=\left(\mathbb{I}-\mathbf{K}_{k}^{e} \mathbf{H}\right) \mathbf{P}_{k}^{f_{e}}
$$

This distribution provides us a natural expression for the proposal distribution. In order to make the estimation of the filtering distribution exact (up to the sampling), each member of the ensemble must be weighted at each instant, $k$, with appropriate weights, $w_{k}^{(i)}$, defined from (13). With a systematic resampling scheme and for high dimensional systems represented on the basis of a very small number of particles the weights simplify as [12]:

$$
w_{k}^{(i)} \propto p\left(\mathbf{y}_{k}^{o} \mid \mathbf{x}_{k}^{(i)}\right), \text { and } \sum_{i=1}^{N} w_{k}^{(i)}=1
$$

The Weighted ensemble Kalman filter (WEnKF) procedure can be simply summarized by the algorithm 1 .

\section{WEnKF assimilation of SLK observations}

In this Section, we present our WEnKF formulation based on the SLK optical flow estimates. In what follows, we detail the dynamical model, the observation model, and the strategy we adopt to incorporate the uncertainties supplied by the SLK estimator. 


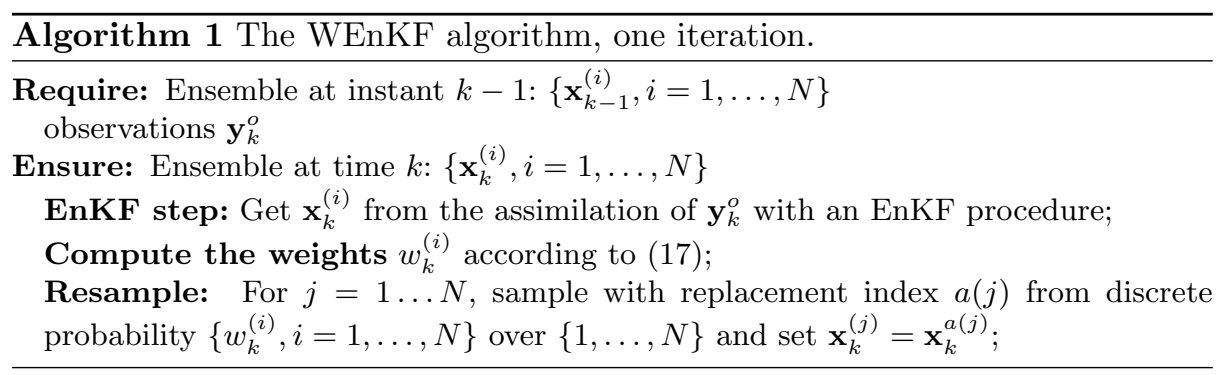

Dynamical model: As in this work we considered only 2D incompressible fluid flows, we will rely for the dynamics on the vorticity-velocity formulation of the Navier-Stokes equation with a stochastic forcing function:

$$
d \xi=-\nabla \xi \cdot \mathbf{v} d t+\nu \Delta \xi d t+\eta d B,
$$

where the state vector $\mathbf{x}=\xi=v_{x}-u_{y}$, represents the vorticity of the velocity field $\mathbf{v}=[u, v]^{T}, \nu$ is the kinematic viscosity and $\eta d B$ is a random forcing term (see following section). The velocity field can be recovered from its vorticity using the Biot-Savart kernel. The numerical simulation of this dynamical model is detailed in [12].

Observation model: The measurements on which we will rely on are set directly as the curl map (i.e. vorticity) of the SLK velocity estimates (4). Assuming the observation is a corrupted version of the true vorticity map (state), we define the observation model as:

$$
\mathbf{y}_{k}=\mathbf{x}_{k}+\gamma_{k}
$$

where $\gamma_{k}$ is a Gaussian random field whose variance is fixed to the spatially varying uncertainties associated to the measurements. These uncertainties are provided by our motion estimator from equation (6) where the expectations have been approached with ensemble empirical mean over the displaced image corresponding to each ensemble members.To mitigate the effect of outliers a Gaussian smoothed version of these variances is considered.

Random fields sampling: To simulate the random forcing term $d \mathbf{B}$ in the dynamics (18) and the random field of the observation model (19), homogeneous Gaussian fields, correlated in space, but uncorrelated in time are used. Their covariance have a general form given by:

$$
Q_{i s o}(\mathbf{r}, \tau)=\mathbb{E}\left[d \mathbf{B}(\mathbf{x}, t) d \mathbf{B}^{T}(\mathbf{x}+\mathbf{r}, t+\tau)\right]=\mathbf{g}_{\lambda}(\mathbf{r}) d t \delta(\tau),
$$

where $\mathbf{g}_{\lambda}(\mathbf{r})$ describes the spatial correlation structure with cutoff parameter $\lambda$. These random fields are in practice sampled in the Fourier domain.

WEnKF implementation: With this dynamics and observation models the WEnKF can be directly implemented as follow. At $k=0$, the ensemble of states $\left\{\mathbf{x}_{0}^{a,(i)}, i=1, \ldots, N\right\}$ is initialized with noisy versions of the SLK vorticity map obtained from the two first images of the sequence. At the current time, the ensemble obtained at the previous measurement instant is propagated through the stochastic state dynamics (18) to generate the forecast ensemble members 
$\mathbf{x}_{k}^{f,(i)}$. The EnKF update is then performed with the new observation in order to sample the proposal distribution. The importance sampling weighting based on the likelihood and a resampling process of the particles with respect to those weights are performed. The empirical mean of the analysis ensemble provides the vorticity estimate at time $k$. The corresponding velocity field is finally obtained from the Biot-Savart law.

Although this direct WEnKF filtering of the SLK vorticity maps does provide good results as we shall see it, the estimation may fail for long range velocities. To overcome this limitation and to further improve the performance of the WEnKF we propose in the next section a multiscale extension of WEnKF.

\section{$5 \quad$ Multiscale SLK-WEnKF filtering}

The idea of multiscale WEnKF consists to provide an improved proposal distribution from velocity measurements at different scales. The update step operates iteratively in an incremental coarse-to-fine way by introducing motion measurements obtained at different scales through the Gaussian smoothing parameter $\lambda_{\ell}$ in (4). More precisely, at scale $\ell \in\left[0, \ell_{f}\right]$ the proposal ensemble is build from successive analysis steps as follow:

$$
\begin{aligned}
& \mathbf{x}_{k}^{a,(i), \ell}=\mathbf{x}_{k}^{f,(i), \ell}+\mathbf{K}_{k}^{e, \ell}\left(\mathbf{y}_{k}^{\ell}+\gamma_{k}^{(i), \ell}-\mathbf{H} \mathbf{x}_{k}^{f,(i), \ell}\right), \\
& \mathbf{x}_{k}^{f,(i), \ell}=\mathbf{x}_{k}^{f,(i), \ell-1}-\overline{\mathbf{x}}_{k}^{a, \ell-1}
\end{aligned}
$$

where the measurements $\mathbf{y}_{k}^{\ell}$ are supplied by the stochastic Lucas and Kanade motion estimates between the backwarped image $\widetilde{I}_{k}^{\ell}=f\left(\mathbf{X}_{k-1}+\sum_{l=0}^{\ell-1} \overline{\mathbf{x}}_{k}^{a, l}, k\right)$ and image $I_{k-1}=f\left(\mathbf{X}_{k-1}, k-1\right)$ within the range of scale $\left[\lambda^{\ell-1}, \lambda^{\ell}\right]$. The quantity $\overline{\mathbf{x}}_{k}^{a, \ell}$ denotes the empirical mean of the analysis ensemble. The initial analysis ensemble is fixed to a null value $\left(\overline{\mathbf{x}}_{k}^{a, \ell_{c}-1}=0\right)$ and the initial forecast is set to the forecast ensemble computed from the dynamics $\left(\mathbf{x}_{k}^{f,(i), 0}=\mathbf{x}_{k}^{f,(i)}\right)$. At each scale, the Gaussian random fields attached to the measurements are drawn with the uncertainties provided by the stochastic Lucas and Kanade formulation computed from the couple of images $\left(\widetilde{I}^{\ell}, I_{k-1}\right)$ and the current analysis ensemble (6). Let us note that compared to the previous single scale filtering where the proposal was based on a single ensemble Kalman update, here several updates associated to different Kalman gains are considered. In the experimental section three successive scales will be considered in such a filtering. The final proposal corresponds to the sum of the analysis ensemble obtained at the different scales: $\mathbf{x}_{k}^{a,(i)}=\sum_{L}^{l} \mathbf{x}_{k}^{a,(i), \ell}$. In the same way as for the previous filter, these ensemble members are then resampled according to the importance weights computed from the likelihood associated to the original couple of images $\left(I_{k-1}, I_{k}\right)$.

\section{Experimental Results and Comparisons}

In this section, we present the results obtained by the application of the single scale and the multiscale WEnKF denoted as $1 \mathrm{~L}-\mathrm{WEnKF}$ and 3L-WEnKF respectively as the latter has been applied on a set of 3 three successive scale ranges. 
Those filters have been compared with state-of-the-art fluid motion estimators $[6,11,14]$ on a sequence of 100 simulated PIV images with a known ground truth corresponding to the numerical simulation (DNS) of a forced $2 \mathrm{D}$ turbulence at Reynolds 3000 available at http: //www.fluid.irisa.fr. Quantitative comparisons with the ground truth in terms of the Root-Mean-Square-Error (RMSE) of vorticity and velocity are both presented in figure 1.

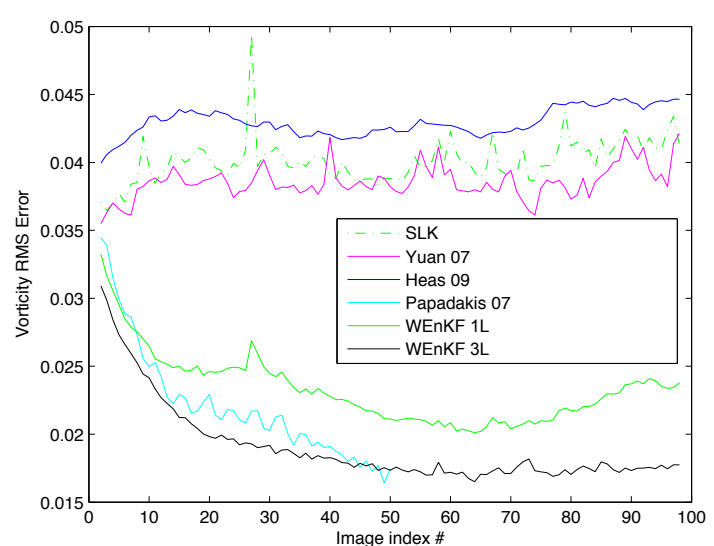

(a)

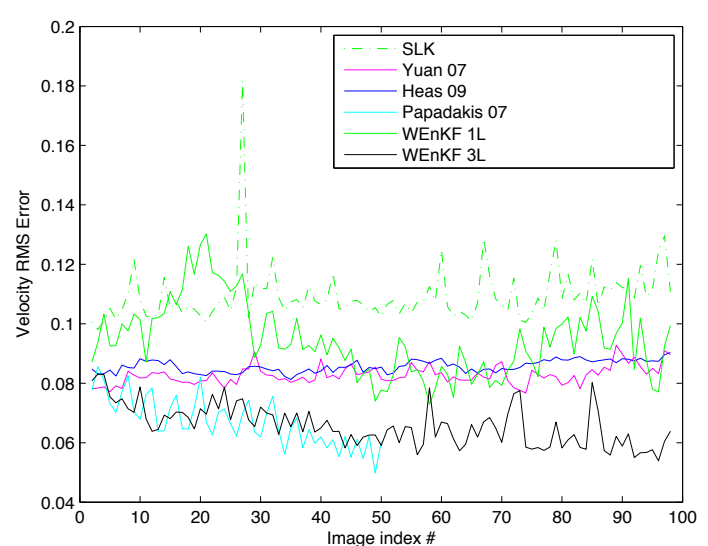

(b)

Fig. 1. Comparison with State of the Art: RMSE in (a) Vorticity (b) Motion field .

As we notice from the fig. 1(a), the RMSE in vorticity of the SLK approach is close to the state of art approaches [14,6] (0.04), though the RMSE values in velocity are higher (fig. 1(b)). The RMSE in vorticity by assimilating the SLK observation through 1L-WEnKF is much lower (0.03), while the error in terms of velocity estimates is close to the approach of Yuan et al. [14]. However, the 3L-WEnKF assimilation shows better results both in terms of vorticity or velocity. These errors are lower than all the fluid motion estimators that have been tested and are at the same level as the errors provided by the batch variationnal assimilation techniques ${ }^{2}$ [11] (which corresponds thus to a smoothing filter as opposed to a recursive filter as in our case).

For a visual comparison we show in fig. 2, the vorticity maps obtained by the different methods for the 50th images of the sequence. The vorticity estimated by the 3L-WEnKF assimilation ( fig. 2 (h)) corresponds to the lowest error.

Our next set of results corresponds to a real world image sequence of a $2 \mathrm{D}$ turbulence generated from the wake of a soap film behind a comb. The flow is visualized through a Schlieren technique at a rate of 2500 frames per second. A typical image of the sequence is shown in figure 3(a) in false color. The

\footnotetext{
$\overline{2}$ The results were unfortunately available only for 50 images.
} 


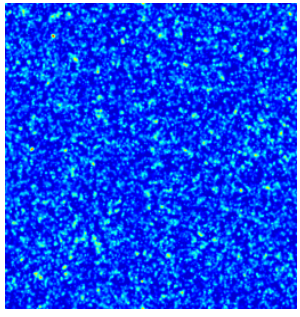

(a)

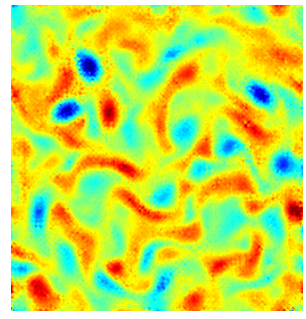

(e)

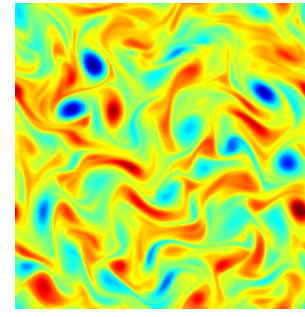

(b)

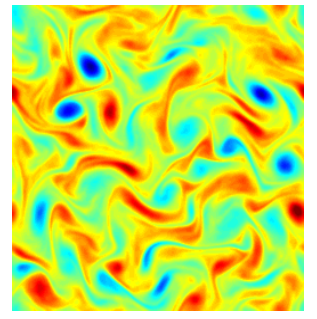

(f)



(c)

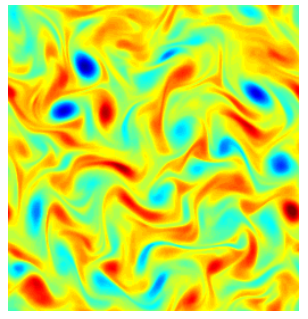

(g)

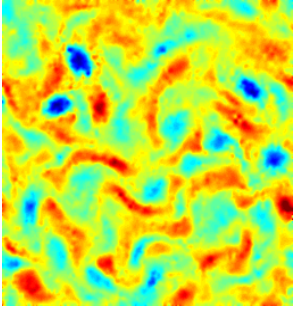

(d)



(h)

Fig. 2. (a) Particle image 50 (b) True vorticity and the estimates of (c) SLK (d) Yuan et al. [14] (e) Heas et. al. [6] (f) Papadakis et al. [11] (g) WEnKF assimilation and (h) 3L-WEnKF assimilation

estimated vorticity maps and velocity fields corresponding to SLK, 1L WEnKF and 3L WEnKF are shown in figs. 3(b), (c) and (d), respectively. We note that though the 1L-WEnKF assimilation of SLK brings out some details at a smaller scale than the SLK measurements, the 3L-WEnKF assimilation recovers even finer details. It is however important to remarks that all those results remains consistent and are close when interpreted at a larger scale.

\section{Conclusion}

In this paper, we have proposed an efficient multiscale extension of the Weighted Ensemble Kalman filter for fluid flow motion estimation problem. This filter is a particle filter relying on a proposal distribution built from the ensemble Kalman filtering mechanism. The particular instance we considered here incorporates measurements issued from a stochastic extension of the Lucas and Kanade estimator.

Acknowledgements The authors acknowledge the support of the French Agence Nationale de la Recherche (ANR), under grant PREVASSEMBLE (ANR-08COSI-012).

\section{References}

1. T. Corpetti, P. Heas, E. Memin, and N. Papadakis. Pressure image asimilation for atmospheric motion estimation. Tellus, 61A:160-178, 2009. 


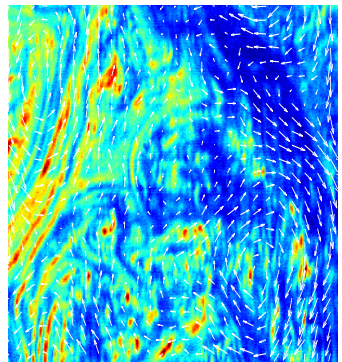

(a)

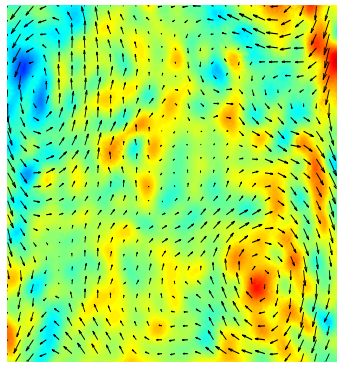

(b)

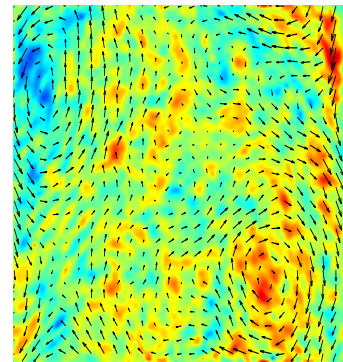

(c)

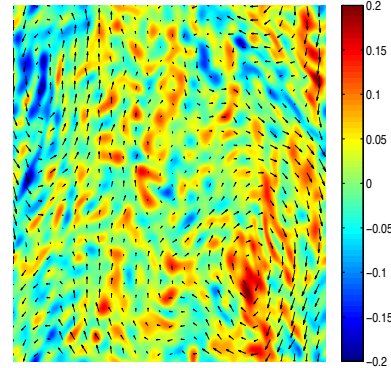

(d)

Fig. 3. Real image sequence of a $2 \mathrm{D}$ turbulent flow of a soap film (a) an image with the $3 \mathrm{~L}$ WEnKF velocity field superimposed; Vorticity maps with their corresponding velocity fields (b) SLK (c) 1L- WEnKF and (d) 3L-WEnKF.

2. T. Corpetti, D. Heitz, G. Arroyo, E. Memin, and A. Santa-Cruz. Fluid experimental flow estimation based on an optical-flow scheme. Experiments in fluids, 40:80-97, 2006.

3. T. Corpetti and E. Memin. Stochastic models for local optical flow estimation. In Int. Conf. on Scale Space and Variational Methods in Computer Vision, SSVM'11, 2011.

4. G. Evensen. Sequential data assimilation with a non linear quasi-geostrophic model using Monte Carlo methods to forecast error statistics. J. Geophys. Res., 99 (C5) (10):143-162, 1994.

5. G. Evensen. The ensemble Kalman filter, theoretical formulation and practical implementation. Ocean Dynamics, 53(4):343-367, 2003.

6. P. Heas, E. Memin, D. Heitz, and P. Mininni. Bayesian selection of scaling laws for motion modeling in images. In Proc. Int. Conf. Computer Vision, 2009.

7. D. Heitz, E. Memin, and C. Schnoerr. Variational fluid flow measurements from image sequences: synopsis and perspectives. Exp. in Fluids, 48(3):369-393, 2010.

8. R.E. Kalman. A new approach to linear filtering and prediction problems. Transactions of the ASME - Journal of Basic Engineering, 82:35-45, 1960.

9. B. Lucas and T. Kanade. An iterative image registration technique with an application to stereovision. In Int. Joint Conf. on Artificial Intel. (IJCAI), pages 674-679, 1981.

10. B. Oksendal. Stochastic differential equations. Spinger-Verlag, 1998.

11. N. Papadakis and E. Memin. An optimal control technique for fluid motion estimation. SIAM Journal on Imaging Sciences, 1(4):343-363, 2008.

12. N. Papadakis, E. Memin, A. Cuzol, and N. Gengembre. Data assimilation with the weighted ensemble kalman filter. Tellus-A, 62(5):673-697, 2010.

13. P. Ruhnau, T. Kohlberger, C. Schnoerr, and H. Nobach. Variational optical flow estimation for particle image velocimetry. Exp.in Fluids, 38:21-32, 2005.

14. J. Yuan, C. Schnoerr, and E. Memin. Discrete orthogonal decomposition and variational fluid flow estimation. J. Mathematical Imaging and Vision, 28(1):6780, 2007. 